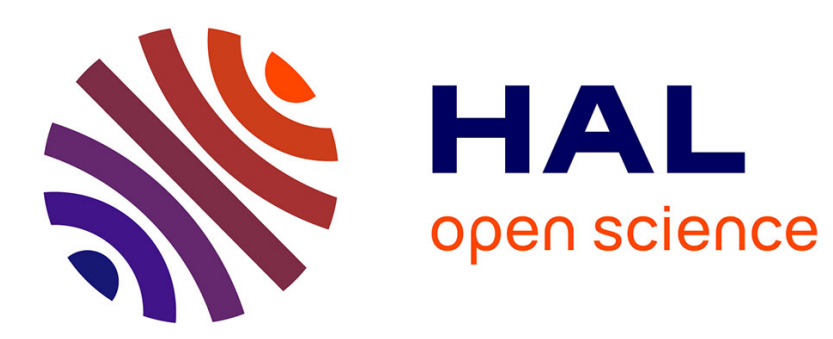

\title{
Effect of embryonic light exposure on laterality and sociality in quail chicks (Coturnix coturnix japonica)
}

Isabelle George, Noémie Lerch, Christelle Jozet-Alves, Sophie Lumineau

\section{To cite this version:}

Isabelle George, Noémie Lerch, Christelle Jozet-Alves, Sophie Lumineau. Effect of embryonic light exposure on laterality and sociality in quail chicks (Coturnix coturnix japonica). Applied Animal Behaviour Science, 2021, 236, pp.105270. 10.1016/j.applanim.2021.105270 . hal-03145313

\section{HAL Id: hal-03145313 \\ https://hal-univ-rennes1.archives-ouvertes.fr/hal-03145313}

Submitted on 12 Mar 2021

HAL is a multi-disciplinary open access archive for the deposit and dissemination of scientific research documents, whether they are published or not. The documents may come from teaching and research institutions in France or abroad, or from public or private research centers.
L'archive ouverte pluridisciplinaire HAL, est destinée au dépôt et à la diffusion de documents scientifiques de niveau recherche, publiés ou non, émanant des établissements d'enseignement et de recherche français ou étrangers, des laboratoires publics ou privés. 
Isabelle George, Noémie Lerch, Christelle Jozet-Alves, Sophie Lumineau. Effect of embryonic light exposure on laterality and sociality in quail chicks (Coturnix coturnix japonica). Applied Animal Behaviour Science, Elsevier, 2021, 236, pp.105270. 〈hal-03145313〉

\section{Authors' post-print}

Editor's version available at the following:

https://dx.doi.org/10.1016/j.applanim.2021.105270

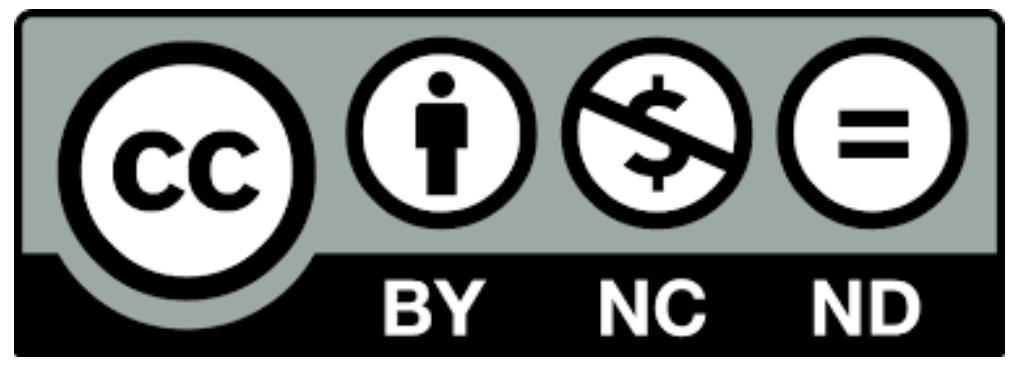




\section{Effect of embryonic light exposure on laterality}

\section{2 and sociality in quail chicks (Coturnix coturnix}

\section{3 japonica)}

4 Isabelle George ${ }^{1, *}$, Noémie Lerch $^{1}$, Christelle Jozet-Alves ${ }^{1,2}$, Sophie Lumineau ${ }^{1}$

$5{ }^{1}$ Univ Rennes, Normandie Univ, CNRS, EthoS (Éthologie animale et humaine) - UMR 6552, F-35000

6 Rennes, France

$7 \quad{ }^{2}$ Normandie Univ, Unicaen, CNRS, EthoS, 14000 Caen, FRANCE

$8 \quad *$ Corresponding author:

9 Isabelle GEORGE

10 UMR 6552 - EthoS

11263 avenue du Général Leclerc

12 Campus de Beaulieu - Bâtiment 25

1335042 Rennes cedex, France

14 isabelle.george@univ-rennes1.fr, +33223236863 


\section{ABSTRACT}

Lateralization is the ascendency of one side of the body or the brain to control and perform motor or perceptual functions. Light exposure during prenatal life can modulate the expression of visual lateralization (strength and direction) in a variety of species, including birds. Individual lateralization provides cognitive advantages, but the function of lateralization at the population level is less obvious. Recent studies support the hypothesis that intra-specific population-level lateralization may favour coordination between asymmetrical individuals during synergistic (cooperative) social interactions. Since cohesion requires a good coordination, one can therefore imagine that a higher alignment of lateralization within a group, by making coordination between individuals easier, may promote social cohesion. Our study investigated the effect of light on laterality and social behaviour in Japanese quail chicks (Coturnix coturnix japonica). We compared chicks coming from eggs exposed to light during incubation to chicks coming from eggs maintained in complete darkness. We expected light-exposed (LE) chicks to be more aligned than dark-incubated (DI) ones and we wanted to test whether the most aligned chicks were also the most coordinated. We assessed laterality, social motivation, synchronization and spatial cohesion within groups of LE and DI chicks. The prenatal light conditions did not affect either laterality or chicks' social behaviour, and there was no turning bias at the group level. An absolute laterality index characterizing the alignment of chicks living together showed that the groups with the highest indices were not necessarily the groups with the greatest synchronization and spatial cohesion. This suggests that light does not consistently induce laterality in Japanese quail chicks and that the alignment of the chicks' turning bias does not influence their social cohesion. Quails are widely used in farm industry and scientific research and better knowledge of the consequences of incubation conditions on their behavioural asymmetries and sociality could help improve their handling and welfare.

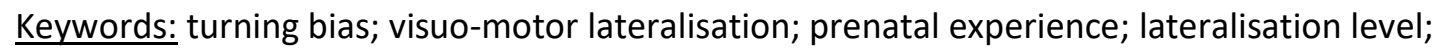
behavioural synchronization; spatial cohesion 


\section{INTRODUCTION}

Laterality is when one side of the body takes advantage over the other one to perform motor or sensory activities (Glick, 1985). It exists in humans and most animal species, including invertebrates (e.g. see Güntürkün et al., 2020; Rogers et al., 2013; Versace and Vallortigara, 2015 for recent reviews). Among behavioural asymmetries, visual lateralization has been widely studied in birds because their eyes are placed laterally and the optic nerves decussate almost completely (Ehrlich and Mark, 1984; Remy and Güntürkün, 1991; Takatsuji et al., 1983; Weidner et al., 1985). This makes them natural "split-brain" models whose eyes and each contralateral hemisphere can be tested independently, which may help in understanding the brain asymmetries that govern lateralization.

Light is known to modulate visual lateralization and hence visually guided behaviours, particularly in birds (e.g. domestic chicks: Casey and Karpinski, 1999; Rogers, 1990, 1982; bobwhite quail: Casey and Lickliter, 1998; pigeons: Güntürkün, 1993; for a review see Chiandetti, 2017). Asymmetrical stimulation of the right eye due to the position of the embryo in the egg (which causes the left eye to be occluded because it is oriented towards the body) is decisive for several forms of visual lateralization (Chiandetti, 2017). In the domestic chick, over-exposure of the right eye to light can reinforce the population bias whereas exposure of the left eye can induce a reversal of this bias. Incubation in the dark can suppress the development of some forms of lateralization at the population level (Rogers, 1990).

Individual lateralization (when each individual favours one side over the other) can give a cognitive advantage, but the function of lateralization at the population level (when most individuals favour the same side) is less obvious (see Vallortigara and Rogers, 2020 for a review of costs and benefits of these two degrees of lateralization). According to some authors, the alignment of individuals' biases may have evolved as an evolutionary stable strategy in which asymmetrical individuals must coordinate their behaviour with that of other asymmetrical individuals (Ghirlanda et al., 2009; 
Ghirlanda et al. (2009), population-level lateralization could be advantageous in intra-specific social interactions by making coordination easier. Thus, synergistic activities could favour individuals with the same lateralization because they can, for instance, have an easier time coordinating physical activities (see also Frasnelli and Vallortigara, 2018). Here we wanted to check whether groups made of individuals with the same lateralization would indeed have an easier time synchronizing their activities and staying cohesive.

Although the domestic chick and the pigeon have been the two most popular models since researchers began studying visual lateralization in birds, more recently Japanese quails have also been studied more extensively (e.g. Casey and Sleigh, 2014; Valenti et al., 2003; Zucca and Sovrano, 2008). Japanese quails have been domesticated for centuries (Cheng et al., 2010). Because of their relatively small size and fast development, they quickly became popular in both farm industry and scientific research. Although quails are difficult to observe in the wild due to their small size and their secretive habits, several studies have shown that, during the breeding season, they live in small family groups or single pairs, in areas where they hardly tolerate other quails (Farris, 1964; Nichols, 1991; Wetherbee, 1961). Japanese quail chicks do not naturally exhibit turning bias and footedness (Casey, 2005; Casey and Sleigh, 2001). However, the chicks, but not the adults, are lateralized in the "pebble floor" task (which consists in distinguishing edible grains from pebbles stuck to the ground) (Valenti et al., 2003). Adult quails, on the other hand, appear to be lateralized in a detour task using a social target (Zucca and Sovrano, 2008) and when they are trained to run across either a left- or a right-turning runway to obtain sexual access to a conspecific of the opposite sex (Gülbetekin et al., 2009, 2007). Recently, by manipulating prenatal visual stimulation after having removed the shell and inner-shell membrane of the egg, Casey and Sleigh (2014) managed to induce individual- and population-level motor lateralization in Japanese quail chicks. Stimulated chicks showed a turning bias that unstimulated chicks did not show. Although embryonic exposure to light is likely to be reduced in wild quails, stimulating embryos with light could be useful to manipulate lateralization, at 
the individual as well as at the population level, and to better understand the link between lateralization and social behaviour.

Here, to test the effect of light on population-level laterality and social behaviour, and to determine whether the alignment of behavioural asymmetries can facilitate social interactions between individuals, we compared the turning bias, temporal synchronisation, spatial cohesion and social motivation of groups of quail chicks coming from eggs that had been either incubated in the dark or temporarily exposed to light before the end of incubation. We expected that exposure to light would create differences in visuo-motor lateralization between exposed and unexposed chicks, and we wanted to see if the most lateralized chicks were also the most coordinated and socially motivated.

\section{MATERIAL AND METHODS}

All the experiments were carried out in accordance with the European Communities Council Directive of 22th September 2010 (2010/63/EU) and were approved by the regional ethics committee (French “Comité Rennais d'Ethique en matière d'Expérimentation Animale / $n^{\circ} 7$ ", permit number: APAFIS\#17476-2018110716226608 v2).

\subsection{Incubation}

We placed commercial fertilized Japanese quail eggs ("Les cailles de Chanteloup", Corps-Nuds, France) in two incubators (Ova-Easy Advance 380, Brinsea). From day 1 of incubation to day 15, incubation parameters were the same for all eggs: $37.7^{\circ} \mathrm{C}, 45 \%$ humidity and a rotation of 45 degrees every 30 minutes, and both incubators were in the dark. After 15 days of incubation, to induce hatching, we changed the parameters of both incubators to $37.7^{\circ} \mathrm{C}, 65 \%$ humidity and no rotation. At the same time, we interchanged half of the eggs of each incubator in order to avoid any incubator bias. From that moment, the eggs of one of the incubators were lit continuously for 41 hours by a "daylight" led $\left(6400^{\circ} \mathrm{K}, 600\right.$ lumens) placed in front of the incubator while the eggs of the second incubator stayed in the dark. After the 41 hours of exposition to light, we again placed all the 
eggs in the dark until hatching on day 17 to 18 , that is within 24 hours after the end of the stimulation. Since light intensity within the incubator was 20-30 Lux, luminous exposure was $2.9 \times 10^{6}$ to $4.4 \times 10^{6}$ Ix.s (for comparison, luminous exposure was $0.7 \times 10^{6}$ to $3.6 \times 10^{6}$ in Rogers, 1982). Japanese quail's eggs have brown spots that may reduce the amount of light that reaches the embryo. We therefore decided to stimulate the eggs longer, and thus started the stimulation earlier, than in the study of Casey and Sleigh (2014) on quails. However, the end of the stimulation was similar to theirs.

\subsection{Maintenance of the chicks}

The day of hatching (day 1, D1), we weighed the chicks, ringed them with one plastic ring on each leg, and placed them in heated plastic cages $(\mathrm{L} 90 \mathrm{x} \mid 40 \mathrm{xH} 30 \mathrm{~cm})$ with a plastic net and wood shavings on the floor. We provided food and water ad libitum. Dark-incubated (DI) and light-exposed (LE) chicks were in the same room but not in the same cages. At post-hatching day 3 (D3), we separated 120 of the chicks that had hatched in $2 \times 10$ groups of six chicks ( $n=60 \mathrm{DI}$ and $60 \mathrm{LE}$ chicks). At this age chicks cannot be sexed so we have constituted the groups without knowing their sex ratio. After 3 weeks, we sexed the birds and found that the sex ratio did not differ between DI and LE birds (DI: females $=36$, males $=23 ;$ LE: females $=31$, males $=27 ;$ Fisher's exact test, $p=0.46$, https://biostatgv.sentiweb.fr/?module=tests/fisher).

On the day of hatching, when we took all the chicks that had hatched, DI chicks ( $n=106)$ were heavier than LE chicks ( $n=104)$ (mean $\pm S D$ : DI chicks' weight $=10.51 \pm 0.93 \mathrm{~g}$, LE chick's weight $=10.26 \pm 0.79 \mathrm{~g}$; Student $t=2.03, d d l=208, p$-value $=0.04)$, but the effect was small (Hedges' $g=(10264-10507) /$ $862.06=0.28 ;$ https: $/ /$ www.socscistatistics.com/effectsize/default3.aspx). Anyway, by mixing chicks of different weights within each experimental group, we constituted groups of DI and LE chicks whose average weights were not significantly different $(n=60$ chicks in each group, $D$ I chicks' weight $=$ $10.43 \pm 0.68 \mathrm{~g}, \mathrm{LE}$ chicks' weight $=10.28 \pm 0.66 \mathrm{~g}$; Student $\mathrm{t}=1.17, \mathrm{ddl}=118, \mathrm{p}=0.24)$. We placed all the six-chick groups in the same room in separate metal cages (L100xI70xH65 cm) equipped with a heating lamp, a feeder and a drinking trough (Figure 1). The different groups could hear but not see 
each other. Room temperature was 20 to $25^{\circ} \mathrm{C}$ and the artificial light followed a L:D rhythm of 12:12.

During the first week of life, we provided green light during the night to enable the chicks to see the feeder and drinking trough if they need to eat or drink. We counterbalanced the positions of the DI and LE chicks' groups in the room in order to avoid a bias due to the position of the cage. We also counterbalanced the positions of the feeder and the drinking trough within the cages. We turned off heating lamps after 15 days. Under natural conditions, quail chicks are able to regulate their body temperature and they become independent by 11 days after hatching, (Mills et al., 1997; Orcutt, Jr. and Orcutt, 1976).

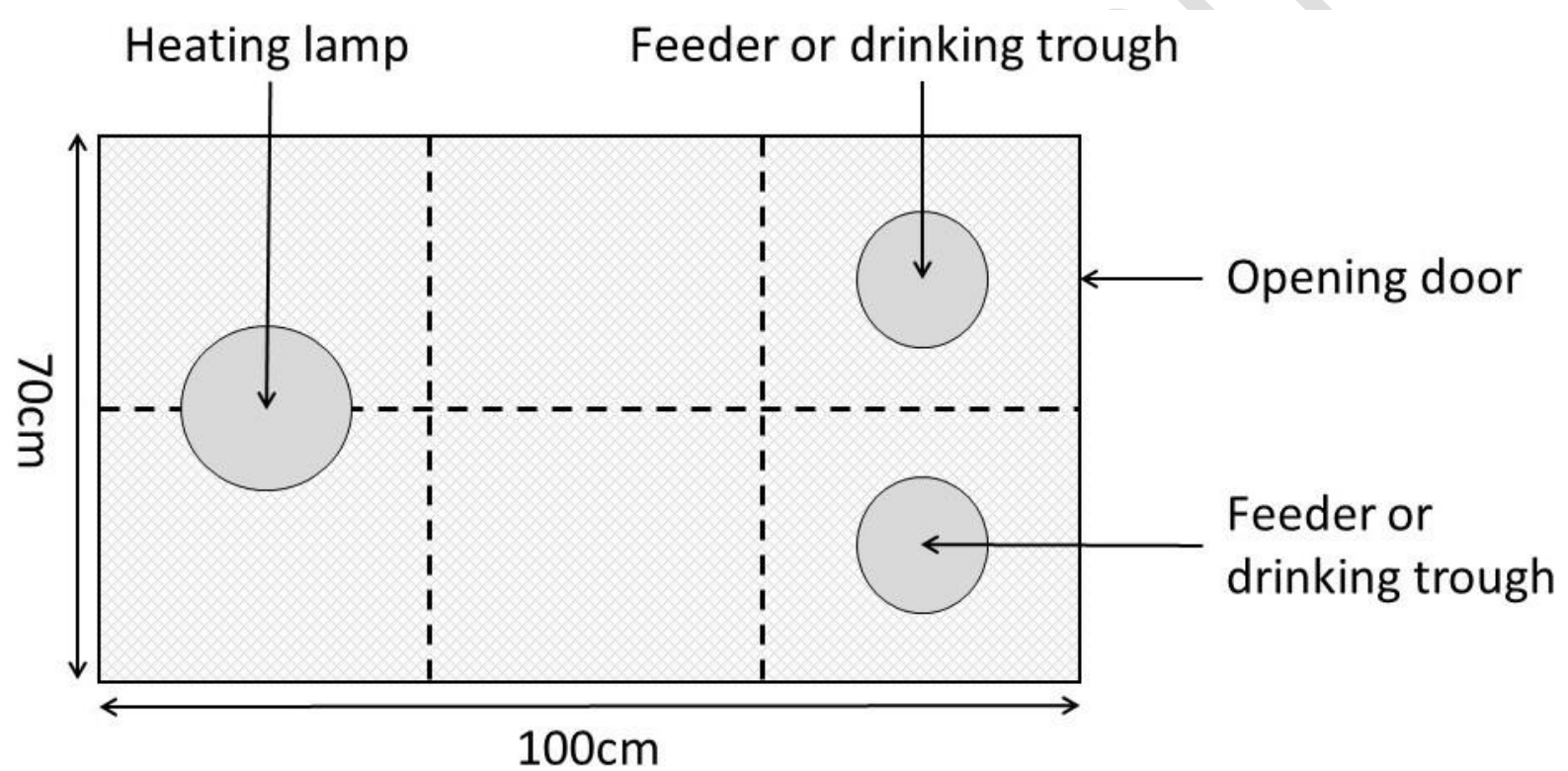

Figure 1: Schematic representation of a home cage. The dotted lines show the virtual boundaries we used to locate the chicks during behavioural observations.

\subsection{Laterality tests}

On D3, once the six-chick groups were formed, we tested the turning bias of the 120 chicks by placing each of them once in a T-maze (Figure 2). The T-maze was in a soundproof room homogeneously lighted. We regularly changed the orientation of the maze in the room $\left(90^{\circ}\right.$ turn each time) so that a quarter of the chicks ( $n=15 \mathrm{DI}$ and $15 \mathrm{LE}$ chicks) passed the test in each of the four positions. At the beginning of each test, the experimenter placed a chick in the longest, stem branch of the T-maze, 
facing the wall, and stood still behind this branch. The chick was free to move in the maze during 3 minutes. The test stopped as soon as the chick turned in one of the distal branches (i.e. both legs were in the branch; see Figure 2) or after 3 minutes, and the experimenter noted which side (left or right) the chick chose. We also tested all the chicks in the same way on D10-11 and D22-23. In order to adapt to their growth, we used a larger maze (Figure 2). We also had to conduct the tests over two days because they moved much less at these ages (Figure 3).

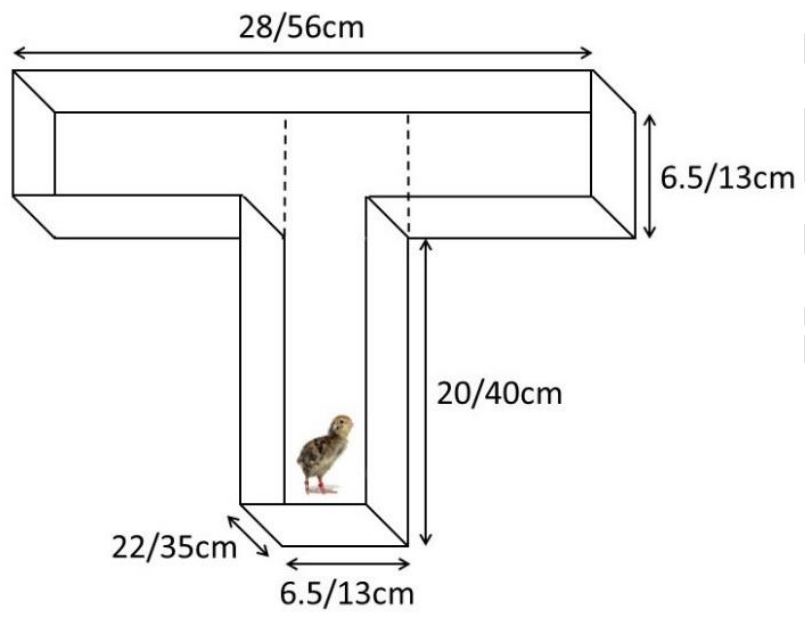

Figure 2: T-maze that we used to test the laterality of the chicks. The smallest dimensions correspond to the size of the maze that we used on D3, and the largest dimensions to the size of the maze that we used on D10-11 and D22-23. We considered that the chick had chosen one branch of the maze when both legs crossed one of the two virtual lines represented here as dotted lines.

\subsection{Behavioural observations}

In order to evaluate the spatial cohesion and the temporal coordination of each group of six chicks, we observed the chicks directly in their home cage with the instantaneous scan-sampling method. Each group was observed 60 times during 12s in two 135-min sessions (one from 8:00 $\mathrm{h}$ to 10:15 $\mathrm{h}$ and one from 15:30 $\mathrm{h}$ to $17: 45 \mathrm{~h}$ ), with an interval of $4 \min 30$ s between two consecutive scans of the same group. We noted the behaviour (resting, moving, exploring, eating, drinking, observing, vocalising, preening and touching another chick) and position of all the chicks in each group. To position the chicks, we virtually divided the cage into six $34 \times 34 \mathrm{~cm}$ squares (Figure 1 ). Behavioural 
176 chicks.

\section{$177 \quad 2.5$ Social motivation test}

178 On D16 and D17, the chicks were isolated one by one in their home cage to test their social motivation. We removed all the chicks from the same home cage and then put them back one by one in this cage. There was only one chick at a time in the home cage and the other chicks were kept together in a room far enough away so that the isolated chick could not hear them. When they lose visual contact with peers, quail chicks usually try to re-establish contact by emitting distress calls that are calls of high social value (Guyomarc'h and Guyomarc'h, 1996). These calls are loud calls that are easily recognisable and they are the only loud calls that are produced when quail chicks are isolated. We noted the latency of the first distress call and the total number of calls during 3 minutes. After these 3 minutes, the isolated chick was removed, placed back with the others and another chick was placed alone in the cage for 3 minutes, until all six chicks had been tested.

This test measures both social motivation and emotivity. However, by leaving the tested chick in the home cage, that is in a familiar environment, we tried to make it feel as safe as possible. This allowed us to avoid an emotional or fear response due to novelty and a supposedly dangerous situation and to thus preferentially measure the social dimension of the chicks' response.

\subsection{Statistical analyses}

We compared the number of DI and LE chicks having turned (left or right) or not in the T-maze, and the number of chicks turning left and right, using Chi-squared tests within (goodness of fit) and between (homogeneity) the experimental conditions, using Microsoft Excel 2019. 
low-energy behaviours (resting, observing, preening and pecking another chick). If, for one day of observation, $A$ is the number of chicks of the same group having high-energy behaviours in one scan " $\mathrm{i}$ ", $\mathrm{R}$ is the number of chicks having low-energy behaviours in the same scan and $\mathrm{N}$ the total number of chicks per group (6) multiplied by the total number of scans per day (60), then: TS= $\sum_{i=1}^{60} \frac{|A i-R i|}{N}$. TS was 1 when all the chicks of the group were having the same type of behaviour (either high- or lowenergy) in all the scans and 0 when half of the chicks were having one type of behaviour while the other half was having the other one. We obtained one TS value per group of 6 chicks and per day of observation. The second index was a spatial cohesion (SC) index. In each scan, there was $\mathrm{n}_{1}$ chicks in the virtual square $1, n_{2}$ in the virtual square $2 \ldots$ and $n_{6}$ in the virtual square 6 . Using these values, we calculated the ratio between the variance and the mean number of chicks per virtual square per scan. This ratio was 6 when all the chicks were in the same square, and 0 when there was one chick in each square. We then averaged the values of the 60 scans to obtain one SC value per group of six chicks and per day of observation. We compared DI and LE chicks' TS and SC indices obtained for all days with permutation tests using Rd_kheradPajouh_renaud to handle nuisance variables and 50000 permutations (R v4.0.0 with permuco package; Frossard and Renaud, 2019; R Core Team, 2020). tests with the latency of the first distress call and the total number of calls as variables ( $R$ v4.0.0; $R$ Core Team, 2020).

Finally, in order to see if there was a correlation between the alignment of laterality and the social cohesion within the groups of 6 chicks, we calculated for every group and for each test day in the Tmaze an absolute laterality index (AbsLI) whose formula was: $|L-R| / L+R$ where $L$ was the number of chicks who turned left and $R$ the number of chicks who turned right. This index was 1 when all the chicks turned on the same side and 0 when half of the chicks turned on one side and the other half on the other side. We kept only the groups in which at least three of the six chicks chose to turn into 
one of the two arms during the test for further analyses. We used AbsLI values to make linear regressions and calculate correlation coefficients with TS and SC indices.

\section{RESULTS}

\subsection{Effect of light exposure}

\subsubsection{Laterality (T-maze) tests}

At D3 and D22-23, there were significantly more chicks that turned left or right than chicks that did not turn (Figure 3A; see Table 1 for statistical results). At D10-11, since many chicks did not turn (54\% of the DI chicks and $43 \%$ of the LE ones), this difference was not significant (Figure $3 \mathrm{~A}$ ).

Whatever the day, there was no within- or between-group difference in the number of chicks turning left and those turning right (Table 1; Figure 3B).
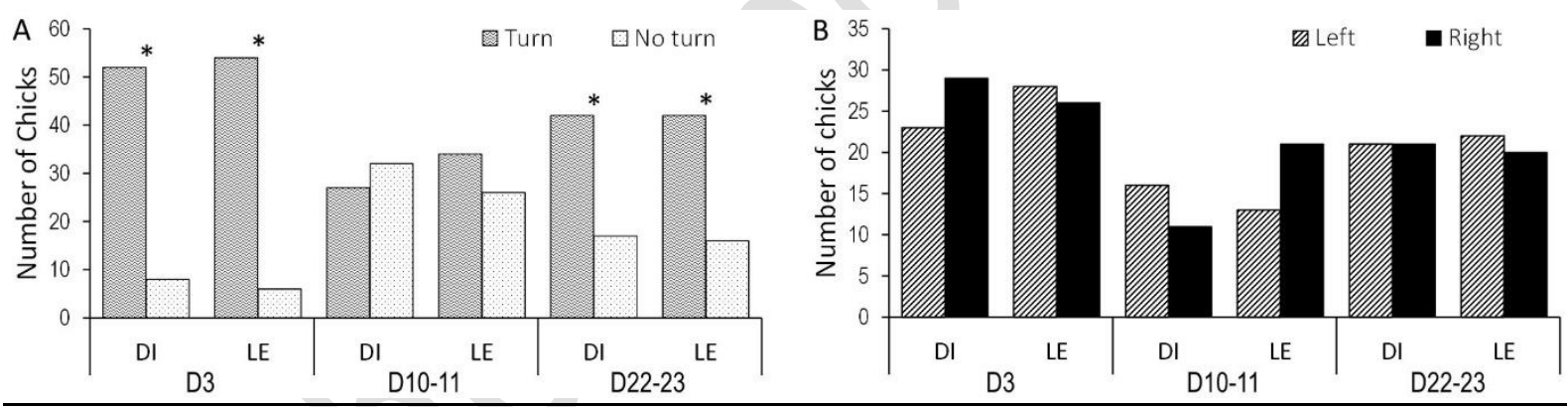

Figure 3: Summed results of the laterality tests at D3, D10-11 and D22-23. (A) Number of chicks that turn or did not turn in the T-maze; (B) Number of chicks that turned left and right. DI: Dark-Incubated chicks; LE: Light-Exposed chicks. * Goodness of fit Chi-squared test, $p<0.001$.

Table 1: Comparisons within and between the experimental conditions ( $\mathrm{DI}$ and LE) of the number of chicks turning or not turning, and turning left or right in the T-maze. Results of goodness of fit (within experimental conditions) and homogeneity (between experimental conditions) Chi-squared tests.

\begin{tabular}{l|ll} 
Turn/ & DI & LE \\
No turn & & DI/LE
\end{tabular}




\begin{tabular}{cccccccccc}
\hline D3 & $X^{2}=32.27$ & $d f=1$ & $p<0.001$ & $X^{2}=38.40$ & $d f=1$ & $p<0.001$ & $X^{2}=0.32$ & $d f=1$ & $p>0.05$ \\
D10-11 & $X^{2}=0.42$ & $d f=1$ & $p>0.05$ & $X^{2}=1.07$ & $d f=1$ & $p>0.05$ & $X^{2}=1.42$ & $d f=1$ & $p>0.05$ \\
D22-23 & $X^{2}=10.59$ & $d f=1$ & $p<0.001$ & $X^{2}=11.65$ & $d f=1$ & $p<0.001$ & $X^{2}=0.02$ & $d f=1$ & $p>0.05$ \\
\hline Left/Right & & Dl & & & LE & & & DI/LE & \\
\hline D3 & $X^{2}=0.69$ & $d f=1$ & $p>0.05$ & $X^{2}=0.07$ & $d f=1$ & $p>0.05$ & $X^{2}=0.62$ & $d f=1$ & $p>0.05$ \\
D10-11 & $X^{2}=0.93$ & $d f=1$ & $p>0.05$ & $X^{2}=1.88$ & $d f=1$ & $p>0.05$ & $X^{2}=2.67$ & $d f=1$ & $p>0.05$ \\
D22-23 & $X^{2}=0.00$ & $d f=1$ & $p>0.05$ & $X^{2}=0.09$ & $d f=1$ & $p>0.05$ & $X^{2}=0.05$ & $d f=1$ & $p>0.05$
\end{tabular}

D: Day; DI: Dark-Incubated chicks; LE: Light-Exposed chicks. Significant results are in bold.

\subsubsection{Temporal synchronisation}

242 Temporal synchronisation decreased with time regardless of the experimental condition (DI/LE:

$243 \mathrm{~F}=0.59, \mathrm{p}=0.45$; Day: $\mathrm{F}=42.12, \mathrm{p}<0.0001$; interaction: $\mathrm{F}=1.06, \mathrm{p}=0.38$ ). The older the chicks were, the

244 less synchronized they were (Figure 4). There was a significant decrease after D4 and temporal

245 synchronization at D15 was significantly lower than at D7 and D9 (Pairwise comparisons using

246 permutation $t$ tests; D4 compared to the other days: $p=0.02$ in all cases; D15 compared to D7 and D9:

$247 \mathrm{p}=0.04$ in both cases). A Bayes factor analysis confirmed a strong effect of time but a negligible effect

248 of the experimental condition and of its interaction with time (supplementary Table 1).

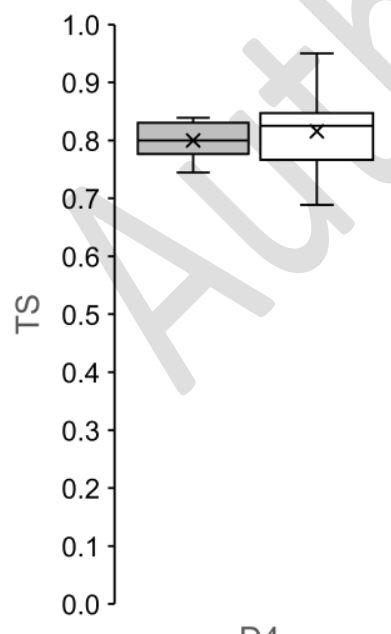

D4

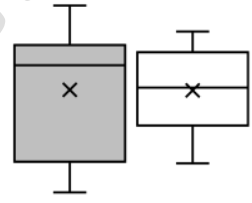

D7 $\square$ DI $\square$ LE
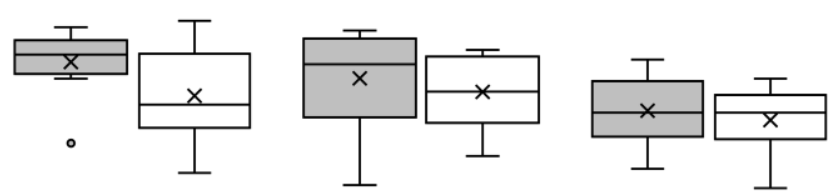

D9

D11

D15

250 Figure 4: Temporal synchronization index (TS) of dark-incubated (DI, grey boxes) and light-exposed

(LE, white boxes) chicks during development ( $D=$ post-hatching day). Boxplot: middle line=median, 


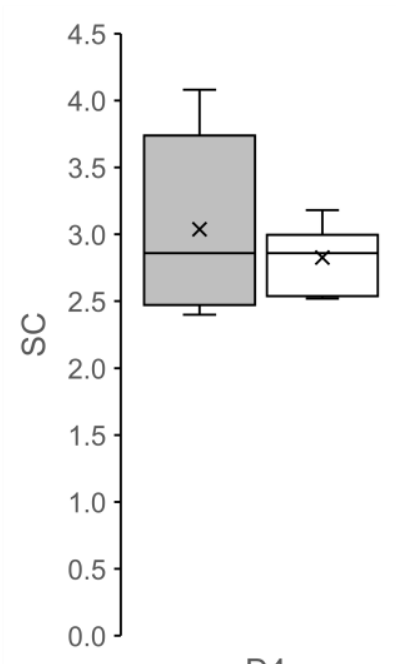

261 range. Cross: mean.

\subsubsection{Spatial cohesion}

D4 mean.

\subsubsection{Social motivation}

lower limit of the box=1st quartile, upper limit of the box=3rd quartile, whiskers=1.5 inter-quartile

After a significant decrease between D4 and D7, spatial cohesion remained relatively stable over time, regardless of the experimental condition (DI/LE: $F=0.00, p=0.98$; Day: $F=5.54, p<0.001$; interaction: $\mathrm{F}=0.87, \mathrm{p}=0.49$; Pairwise comparisons using permutation $\mathrm{t}$ tests; $\mathrm{D} 4$ significantly different of all other days except D15: $\mathrm{p}=0.02$ for D7, D9 and D11 and 0.30 for D15; no other significant difference; Figure 5). Again, a Bayes factor analysis confirmed an effect of time but a negligible effect of the experimental condition and of its interaction with time (supplementary Table 1).

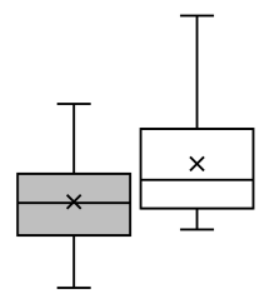

D7
口DI QLE
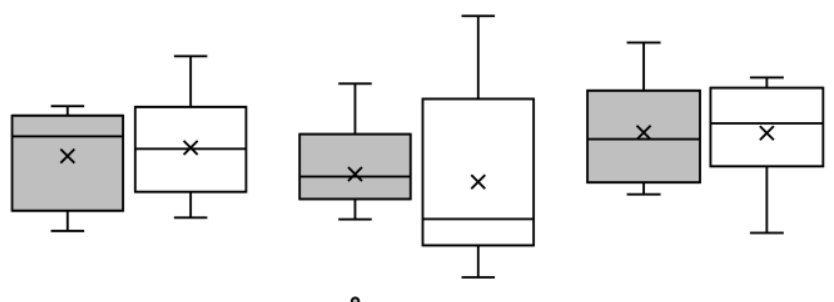

D9

Figure 5: Spatial cohesion index (SC) of dark-incubated (DI, grey boxes) and light-exposed (LE, white boxes) chicks during development ( $D=$ post-hatching day). Boxplot: middle line=median, lower limit of the box=1st quartile, upper limit of the box=3rd quartile, whiskers=1.5 inter-quartile range. Cross:

There was no difference in social motivation between the two experimental conditions as measured by the latency before the first distress call (Mann-Whitney $W=1757.5, n_{D I}=59, n_{L E}=60, p=0.95$ ) or the total number of calls (Mann-Whitney $U=1625.5, n_{D I}=59, n_{L E}=60, p=0.44$ ) (Figure 6). 

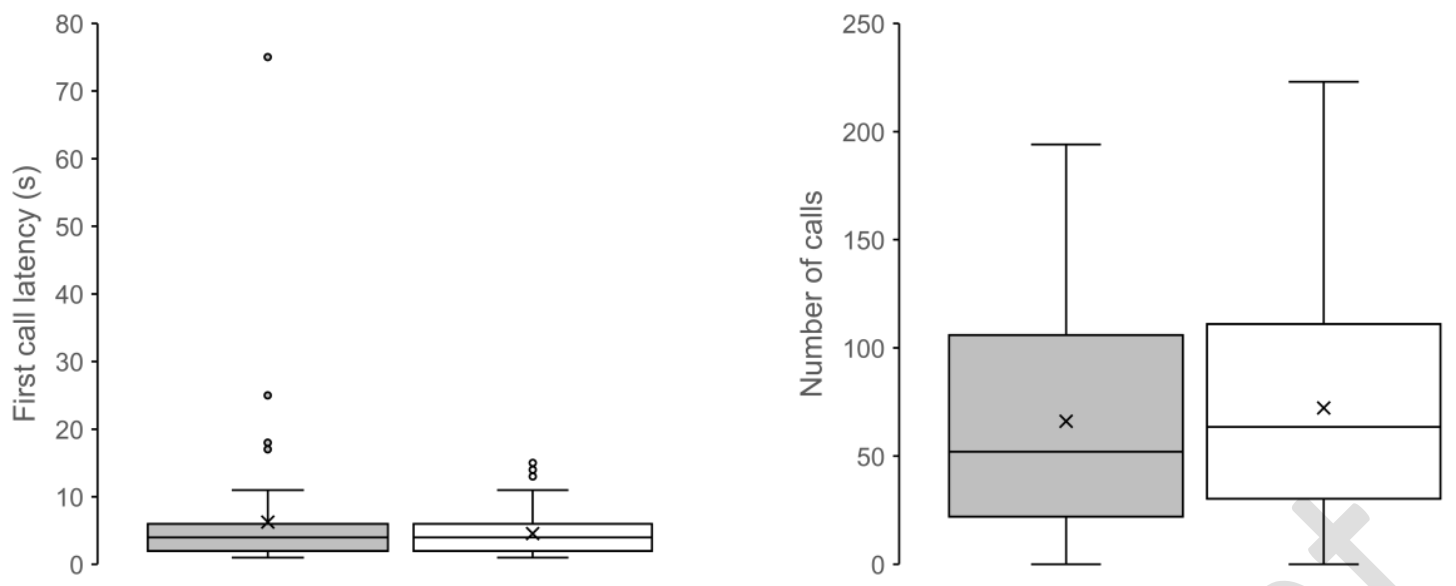

Figure 6: (A) Latency before the first distress call (s) and (B) total number of calls of dark-incubated

271 (DI, grey boxes) and light-exposed (LE, white boxes) chicks during the social motivation test. For better readability, chicks that did not vocalize ( $n=2 \mathrm{DI}$ and $\mathrm{n}=2 \mathrm{LE}$ chicks), and therefore had a latency score of $180 \mathrm{~s}$, were excluded from the latency graph. Boxplot: middle line=median, lower limit of the box=1st quartile, upper limit of the box=3rd quartile, whiskers=1.5 inter-quartile range. Crosses: mean.

\subsection{Correlation between laterality alignment and social cohesion}

277 The AbsLl index measured the extent to which the chicks living in the same cage turned in the same

278 direction in the T-maze test. Whatever the day, there was no correlation between AbsLI and

279 temporal synchronisation or spatial cohesion (Figure 7). This means that the most aligned chicks were not necessarily the most synchronized and cohesive. Since social motivation was only measured once in each chick, we could not test the correlation between AbsLI and the number and latency of distress calls. 

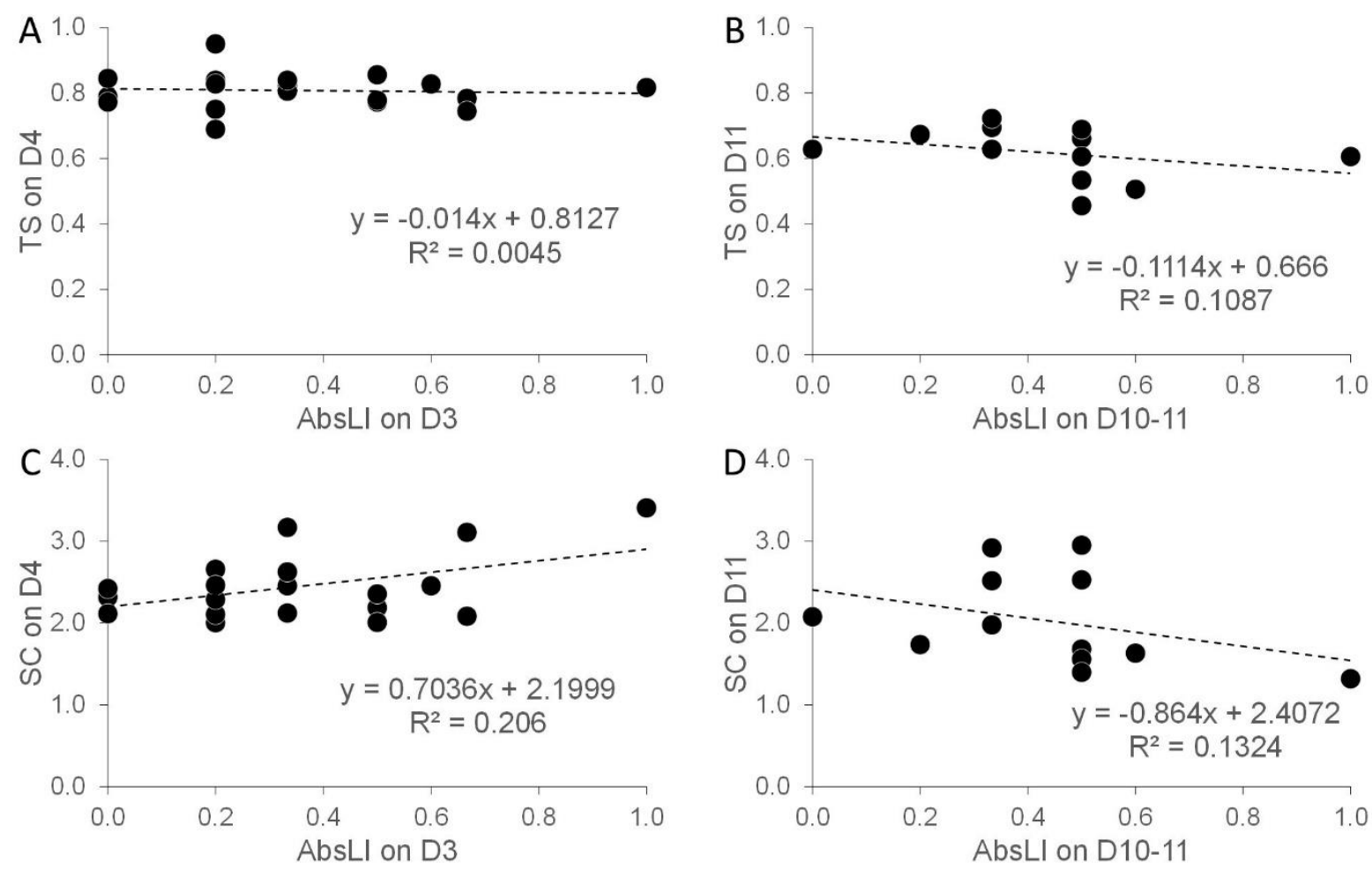

Figure 7: Correlations between AbsLI and $(\mathrm{A}, \mathrm{B})$ temporal synchronization $(\mathrm{TS})$ and $(\mathrm{C}, \mathrm{D})$ spatial

cohesion (SC), at $(A, C) D 3-4$ and $(B, D)$ D10-11. Dotted line: linear regression.

\section{DISCUSSION}

By comparing laterality (at the group level and as measured by turning bias) and social behaviour of groups of chicks exposed or not to light at the end of incubation, we studied both the effect of light on the development and alignment of lateralization and the link between laterality and sociality.

Since the degree, the direction and the alignment of lateralization of some visually-guided

behaviours can be modulated by the amount of light received during incubation in a variety of species such as zebra fish, topminnows, pigeons and domestic chicks (see Chiandetti, 2017 for a review), we expected light exposure to influence the alignment of visuo-motor lateralization and to create a difference between our two experimental conditions. However, our protocol of exposure to light during incubation had no effect either on the alignment of turning bias or on spatial cohesion, temporal synchronisation and social motivation of our groups of chicks. 
Exposure to light during incubation had no significant effect on chicks' visuo-motor laterality in the Tmaze test. Yet, in the domestic hen and the bobwhite quail, two species that are closely related to the Japanese quail, exposure to light before hatching can reinforce visual (Rogers, 1982) and motor (Casey and Karpinski, 1999; Casey and Lickliter, 1998) lateralization at the population level. This effect is thought to be due to the position of the embryo in the egg: in the egg, the embryo's body occludes the left eye and only the right eye can receive light. Since the avian visual system crosses almost completely, the left hemisphere is therefore more stimulated than the right one (Rogers, 1990). Occluding the right eye and stimulating the left one (therefore the right hemisphere) can reverse the group bias (Casey and Karpinski, 1999; Casey and Lickliter, 1998; Rogers, 1990). Thus, asymmetrical light stimulation in the egg can create a bias. Here, we observed no group turning bias in our chicks, even after exposing them to light during incubation. This suggests that light stimulation through the shell does not modulate the particular visuo-motor lateralization that we measured in the Japanese quail. Casey and Sleigh (2014) recently obtained an effect of light during incubation, but they had to open the eggshell and to take out the head of the chick to directly stimulate one of its eyes. It is therefore possible that our light stimulation was not strong enough to play a role in the development of visuo-motor asymmetries. In the domestic hen, a stimulation of 250 to 350 lux of the egg (which corresponds to values as low as 25 to 35 lux for the embryo) is enough to obtain an effect on visual lateralization (Rogers, 1990). But, while hens' eggshells are white, those of Japanese quail have brown spots that may reduce the amount of light that reaches the embryo. Light stimulation did have an effect though: dark-incubated chicks were significantly heavier than light-exposed chicks on the day of hatching. This weight difference may be because light-exposed chicks began to hatch a bit earlier (no more than 9 hours) than dark-incubated chicks. Previous research has shown that exposing eggs to white light can alter the time of hatching (Narahari et al., 1988). This happens especially when light stimulation is too strong (Fairchild and Christensen, 2000; Siegel et al., 1969). Considering this, it might be possible that in our study an excess of light stimulation has made 
hatching earlier in the light-exposed group, which might have somehow disrupted the full development of lateralization.

Another explanation could be that we made our stimulation outside the sensitive period of the Japanese quail. In the domestic chick, the embryos need to be exposed to light during the last two days of incubation to obtain an effect. Although two hours of stimulation are sufficient to create a group bias, stimulation has to last between 2.5 to 6 hours to consolidate this bias, so that it does not change if the other eye is stimulated (Rogers, 1990). More recently, an early sensitive period has been discovered in the domestic chick: light exposure occurring well before the development of a functional visual system also plays a role in the development of visual lateralization (Chiandetti et al., 2013; Chiandetti and Vallortigara, 2019). Since no data about sensitive periods to light (either late or early) are available for the Japanese quail, it remains to be determined whether our stimulation was within or outside a light-sensitive period.

To determine individual lateralization, you have to test each individual several times, and to determine population-level lateralization, you have to test many individuals at least once. Because quail chicks grow very fast, our logistical conditions did not allow us to repeat the laterality tests enough times in a short period of time to determine individual lateralization during the first days of life. We therefore decided to focus on laterality at the population-level by testing more chicks but only once. Even if there is no evidence of a motor population bias in Japanese quail chicks, Casey

340 (2005) showed that about $50 \%$ of the individuals show a turning bias (either to the left or to the 341 right, in equal proportion). Although there was no turning bias at the group level, it is therefore 342 possible that the number of individually lateralized chicks differed between the two experimental groups. Moreover, lateralization is task-specific. We therefore cannot exclude that chicks would have shown a group bias in other tasks such as the pebble floor task, for which a transitory visual lateralization has already been evidenced (Valenti et al., 2003). 
Exposure to light had no effect on social cohesion and motivation either. It has also been shown that exposure of the embryo to light has no effect on dispersal in domestic chicks (Wichman et al., 2009). However, when domestic chicks are exposed to light during incubation, they tend to be more aggressive and to peck more at conspecifics than dark-incubated chicks (Rogers, 1982). Moreover, whereas dark-incubated chicks peck more at unfamiliar than at familiar individuals, light-exposed chicks show no preference (Riedstra and Groothuis, 2004). Since the two eyes do not play the same role in social recognition (Rogers and Andrew, 2002; Vallortigara and Andrew, 1994), this lightinduced effect on the distinction between familiar and unfamiliar individuals is likely due to an alteration of the way each hemisphere process visual information. However, no effect of light exposure during incubation was shown on some social behaviours such as individual recognition or choice behaviour between familiar and unfamiliar chicks (Deng and Rogers, 2002). Since these behaviours are more influenced by postnatal light experience (Deng and Rogers, 2002), we might have obtained different results by manipulating exposure to light after hatching.

Regardless of prenatal treatment, we observed a decrease in chicks' temporal synchronisation.

Chicks are not able to regulate their body temperature before 11 days of life (Orcutt, Jr. and Orcutt, 1976). To keep warm, they thus sleep close from each other, which requires them to synchronize their activities (Formanek et al., 2011). Then, they become more and more independent with time and they show less and less social motivation (François et al., 1998). This could explain the decrease that we observed in our study. Spatial cohesion, on the other hand, remained relatively stable over time. This means that chicks stayed relatively close from each other while doing various activities. This might be explained by the fact that they spent a lot of time resting under the heater at the 367 beginning of the experiment, and then spent more time close to the feeder and the drinking trough 368 (without necessarily eating or drinking). In large aviaries (25-30 $\left.\mathrm{m}^{2}\right)$ reproducing semi-natural 369 conditions, spatial cohesion decreases between the age of 4 and 15 days (Lumineau et al., 2001). Our home cages might therefore have been too small for the chicks to disperse. It could also have something to do with the absence of any visual barriers in the home cage, since opaque barriers 
appear to be important for the development of spatial cognition and its lateralization in the domestic chick (Freire and Rogers, 2005). In addition, as they had nowhere to hide, there may have been no incentive for the chicks to move away from other chicks.

Finally, by using an index that could measure the degree of alignment of chicks independently of prenatal treatment, we could check whether there was a correlation between group lateralization and social cohesion (i.e. temporal synchronization and spatial cohesion). The results showed that chicks that were more aligned were not necessarily better synchronized and more cohesive. This suggests that there may be no modulation of social behaviour by group lateralization in the Japanese quail. In a complementary way, Versace et al. (2020) have just shown that social environment can modulate individual- but not population-level lateralization in fruit flies (Drosophila melanogaster).

The link between lateralization and social interactions is therefore probably not so straightforward and more studies are definitely needed to better understand it. The Japanese quail could be useful to explore this issue further, and, since it is a domestic species, and although our study is primarily fundamental, such studies could also help to better understand the consequences of domestication and to improve handling and welfare of species used in laboratory and farm industries. Respect for animal welfare is a major issue in farm industries. In animal husbandry, in order to withstand high densities, animals must be more socially tolerant and less emotive. Understanding the mechanisms for the development of sociality and emotivity is therefore an essential prerequisite for the adaptation of animals to farming conditions.

\section{ACKNOWLEDGMENTS}

We thank Catherine Blois-Heulin, Cécilia Houdelier and Emmanuel de Margerie for their advice, Marion Charrier for her help with experiments, Virginie Durier, Nathalie George and Matthias Rousseau for helping us analyse the data, and Céline Nicolle for taking care of the birds. 
None

\section{REFERENCES}

401

402

403

404

405

406

407

408

409

410

411

412

413

414

415

416

417

418

419

420

421

422

423

424

425

426

427

428

429

430

431

432

433

Casey, M.B., 2005. Asymmetrical hatching behaviors: The development of postnatal motor laterality in three precocial bird species. Dev. Psychobiol. 47, 123-135. https://doi.org/10.1002/dev.20078

Casey, M.B., Karpinski, S., 1999. The Development of Postnatal Turning Bias is Influenced by Prenatal Visual Experience in Domestic Chicks (Gallus gallus). Psychol. Record 49.

Casey, M.B., Lickliter, R., 1998. Prenatal visual experience influences the development of turning bias in bobwhite quail chicks (Colinus virginianus). Dev. Psychobiol. 32, 327-338. https://doi.org/10.1002/(SICI)1098-2302(199805)32:4<327::AID-DEV7>3.0.CO;2-P

Casey, M.B., Sleigh, M.J., 2014. Prenatal visual experience induces postnatal motor laterality in Japanese quail chicks (Coturnix coturnix japonica). Dev. Psychobiol. 56, 489-497. https://doi.org/10.1002/dev.21116

Casey, M.B., Sleigh, M.J., 2001. Cross-species investigations of prenatal experience, hatching behavior, and postnatal behavioral laterality. Dev. Psychobiol. 39, 84-91. https://doi.org/10.1002/dev.1032

Cheng, K.M., Bennett, D.C., Mills, A.D., 2010. The Japanese Quail, in: Hubrecht, R., Kirkwood, J. (Eds.), The UFAW Handbook on the Care and Management of Laboratory and Other Research Animals. John Wiley \& Sons, Ltd, pp. 655-673. https://doi.org/10.1002/9781444318777.ch42

Chiandetti, C., 2017. Manipulation of Strength of Cerebral Lateralization via Embryonic Light Stimulation in Birds, in: Rogers, L.J., Vallortigara, G. (Eds.), Lateralized Brain Functions: Methods in Human and Non-Human Species, Neuromethods. Springer, New York, NY, pp. 611631. https://doi.org/10.1007/978-1-4939-6725-4_19

Chiandetti, C., Galliussi, J., Andrew, R.J., Vallortigara, G., 2013. Early-light embryonic stimulation suggests a second route, via gene activation, to cerebral lateralization in vertebrates. Sci. Rep. 3, 2701. https://doi.org/10.1038/srep02701

Chiandetti, C., Vallortigara, G., 2019. Distinct effect of early and late embryonic light-stimulation on chicks' lateralization. Neuroscience 414, https://doi.org/10.1016/j.neuroscience.2019.06.036

Deng, C., Rogers, L.J., 2002. Social recognition and approach in the chick: lateralization and effect of visual experience. Anim. Behav. 63, 697-706.

Ehrlich, D., Mark, R., 1984. An atlas of the primary visual projections in the brain of the chick Gallus gallus. J. Comp. Neurol. 223, 592-610. https://doi.org/10.1002/cne.902230410

Fairchild, B.D., Christensen, V.L., 2000. Photostimulation of turkey eggs accelerates hatching times without affecting hatchability, liver or heart growth, or glycogen content1. Poultry Sci. 79, 1627-1631. https://doi.org/10.1093/ps/79.11.1627 
Farris, H.E., 1964. Behavioral development, social organization, and conditioning of courting behavior in the Japanese quail Coturnix coturinix japonica. Michigan State University. https://doi.org/10.25335/M55D8NT88

Formanek, L., Richard-Yris, M.-A., Houdelier, C., Lumineau, S., 2011. Rhythmic birds show a better social integration than arrhythmic birds. Chronobiol. Int. 28, 48-57. https://doi.org/10.3109/07420528.2010.532264

François, N., Mills, A.D., Faure, J.M., 1998. Place preferences of Japanese quail given a permanent choice between a social or a non-social but enriched situation. Behav. Processes 43, 163-170. https://doi.org/10.1016/S0376-6357(98)00010-2

Frasnelli, E., Vallortigara, G., 2018. Individual-Level and Population-Level Lateralization: Two Sides of the Same Coin. Symmetry 10, 739. https://doi.org/10.3390/sym10120739

Freire, R., Rogers, L.J., 2005. Experience-induced modulation of the use of spatial information in the domestic chick. Animal Behaviour 69, 1093-1100. https://doi.org/10.1016/j.anbehav.2004.09.009

Frossard, J., Renaud, O., 2019. permuco: Permutation Tests for Regression, (Repeated Measures) ANOVA/ANCOVA and Comparison of Signals.

Ghirlanda, S., Frasnelli, E., Vallortigara, G., 2009. Intraspecific competition and coordination in the evolution of lateralization. Phil. Trans. R. Soc. B 364, 861-866. https://doi.org/10.1098/rstb.2008.0227

Ghirlanda, S., Vallortigara, G., 2004. The evolution of brain lateralization: a game-theoretical analysis of population structure. Proc. R. Soc. Lond. B 271, 853-857. https://doi.org/10.1098/rspb.2003.2669

Glick, S.D. (Ed.), 1985. Cerebral Lateralization in Nonhuman Species. Academic Press. https://doi.org/10.1016/B978-0-12-286480-3.X5001-0

Gülbetekin, E., Güntürkün, O., Dural, S., Çetinkaya, H., 2009. Visual asymmetries in Japanese quail (Coturnix japonica) retain a lifelong potential for plasticity. Behav. Neurosci. 123, 815-821. https://doi.org/10.1037/a0016406

Gülbetekin, E., Güntürkün, O., Dural, S., Çetinkaya, H., 2007. Asymmetry of visually guided sexual behaviour in adult Japanese quail (Coturnix japonica). Laterality 12, 321-331. https://doi.org/10.1080/13576500701307080

Güntürkün, O., 1993. The ontogeny of visual lateralization in pigeons. German J. Psychol. 17, $276-287$.

Güntürkün, O., Ströckens, F., Ocklenburg, S., 2020. Brain Lateralization: A Comparative Perspective. Physiol. Rev. 100, 1019-1063. https://doi.org/10.1152/physrev.00006.2019

Guyomarc'h, J.-C., Guyomarc'h, C., 1996. Vocal communication in European quail; Comparison with Japanese quail. Comptes rendus de l'Académie des sciences. Série III, Sciences de la vie 319, 827-834.

Lumineau, S., Guyomarc'h, C., Richard, J.-P., 2001. Ultradian Rhythm of Activity in Japanese Quail Groups under Semi-Natural Conditions during Ontogeny: Functional Aspects and Relation to Circadian Rhythm. Biol. Rhythm Res. 32, 373-400. https://doi.org/10.1076/brhm.32.3.373.1339

Mills, A.D., Crawford, L.L., Domjan, M., Faure, J.M., 1997. The behavior of the japanese or domestic quail Coturnix japonica. Neurosci. Biobehav. Rev. 21, 261-281. https://doi.org/10.1016/S0149-7634(96)00028-0

Narahari, D., Mujeer, K.A., Thangavel, A., Ramamurthy, N., Viswanathan, S., Mohan, B., Muruganandan, B., Sundararasu, V., 1988. Traits influencing the hatching performance of Japanese quail eggs. British Poult. Sci. 29, 101-112. https://doi.org/10.1080/00071668808417031

Nichols, C.R., 1991. A comparison of the reproductive and behavioural differences in feral and domestic Japanese quail. University of British Columbia. https://doi.org/10.14288/1.0098657

Orcutt, Jr., F.S., Orcutt, A.B., 1976. Nesting and Parental Behavior in Domestic Common Quail. The Auk 93, 135-141. 
R Core Team, 2020. R: A Language and Environment for Statistical Computing. R Foundation for Statistical Computing, Vienna, Austria.

Remy, M., Güntürkün, O., 1991. Retinal afferents to the tectum opticum and the nucleus opticus principalis thalami in the pigeon. Journal of Comparative Neurology 305, 57-70. https://doi.org/10.1002/cne.903050107

Riedstra, B., Groothuis, T.G.G., 2004. Prenatal light exposure affects early feather-pecking behaviour in the domestic chick. Anim. Behav. 67, 1037-1042. https://doi.org/10.1016/j.anbehav.2003.10.009

Rogers, L.J., 1990. Light input and the reversal of functional lateralization in the chicken brain. Behav. Brain Res. 38, 211-221. https://doi.org/10.1016/0166-4328(90)90176-F

Rogers, L.J., 1982. Light experience and asymmetry of brain function in chickens. Nature 297, 223-225. https://doi.org/10.1038/297223a0

Rogers, L.J., Andrew, R.J. (Eds.), 2002. Comparative vertebrate lateralization. Cambridge University Press, New York.

Rogers, L.J., Vallortigara, G., Andrew, R.J., 2013. Divided brains: The biology and behaviour of brain asymmetries, Divided brains: The biology and behaviour of brain asymmetries. Cambridge University Press, New York, NY, US. https://doi.org/10.1017/СBO9780511793899

Siegel, P.B., Isakson, S.T., Coleman, F.N., Huffman, B.J., 1969. Photoacceleration of development in chick embryos. Comp. Biochem. Physiol. 28, 753-758. https://doi.org/10.1016/0010406X(69)92108-2

Takatsuji, K., Ito, H., Masai, H., 1983. Ipsilateral retinal projections in Japanese quail, Coturnix coturnix japonica. Brain Res. Bull. 10, 53-56. https://doi.org/10.1016/0361-9230(83)90074-6

Valenti, A., Sovrano, V.A., Zucca, P., Vallortigara, G., 2003. Visual lateralisation in quails (Coturnix coturnix). Laterality: Asymmetries of Body, Brain and Cognition 8, 67-78. https://doi.org/10.1080/713754470

Vallortigara, G., 2006. The evolutionary psychology of left and right: Costs and benefits of lateralization. Dev. Psychobiol. 48, 418-427. https://doi.org/10.1002/dev.20166

Vallortigara, G., Andrew, R.J., 1994. Differential involvement of right and left hemisphere in individual recognition in the domestic chick. Behavioural Processes 33, 41-57. https://doi.org/10.1016/0376-6357(94)90059-0

Vallortigara, G., Rogers, L.J., 2020. A function for the bicameral mind. Cortex 124, 274-285. https://doi.org/10.1016/j.cortex.2019.11.018

Vallortigara, G., Rogers, L.J., 2005. Survival with an asymmetrical brain: advantages and disadvantages of cerebral lateralization. Behav. Brain Sci. 28, 575-589. https://doi.org/10.1017/S0140525X05000105

Versace, E., Caffini, M., Werkhoven, Z., de Bivort, B.L., 2020. Individual, but not population asymmetries, are modulated by social environment and genotype in Drosophila melanogaster. Scientific Reports 10, 4480. https://doi.org/10.1038/s41598-020-61410-7

Versace, E., Vallortigara, G., 2015. Forelimb preferences in human beings and other species: multiple models for testing hypotheses on lateralization. Front. Psychol. 6. https://doi.org/10.3389/fpsyg.2015.00233

Weidner, C., Repérant, J., Miceli, D., Haby, M., Rio, J.P., 1985. An anatomical study of ipsilateral retinal projections in the quail using radioautographic, horseradish peroxidase, fluorescence and degeneration techniques. Brain Res. 340, 99-108. https://doi.org/10.1016/00068993(85)90778-4

Wetherbee, D.K., 1961. Investigations in the Life History of the Common Coturnix. Amer. Midland Naturalist 65, 168-186. https://doi.org/10.2307/2423011

Wichman, A., Rogers, L.J., Freire, R., 2009. Visual lateralization and development of spatial and social spacing behaviour of chicks (Gallus gallus domesticus). Behavioural Processes 81, 14-19. https://doi.org/10.1016/j.beproc.2008.12.006 
Zucca, P., Sovrano, V.A., 2008. Animal lateralization and social recognition: Quails use their left visual hemifield when approaching a companion and their right visual hemifield when approaching a stranger. Cortex 44, 13-20. https://doi.org/10.1016/j.cortex.2006.01.002 
540 Temporal synchronisation

541

542 [1] Experimental condition (DI/LE) + Cage: $0.3211489 \pm 0.86 \%$

543 [2] Time (Day) + Cage: $3.81146 e+16 \pm 0.63 \%$

544 [3] Experimental condition (DI/LE) + Time (Day) + Cage: 1.611854e+16 $\pm 1.12 \%$

545 [4] Experimental condition (DI/LE) + Time (Day) + Experimental condition (DI/LE):Time (Day) + Cage:

546 $4.681978 \mathrm{e}+15 \pm 4.07 \%$

547 Against denominator: Temporal Synchronisation Cage

548

549 Spatial cohesion

550

551 [1] Experimental condition (DI/LE) + Cage: $0.279752 \pm 0.86 \%$

552 [2] Time (Day) + Cage: $129.6506 \pm 0.45 \%$

553 [3] Experimental condition (DI/LE) + Time (Day) + Cage: $37.23607 \pm 1.27 \%$

554 [4] Experimental condition (DI/LE) + Time (Day) + Experimental condition (DI/LE):Time (Day) + Cage:

555 $9.711455 \pm 6.99 \%$

556 Against denominator: Spatial Cohesion Cage

557

558

Bayes factor type: BFlinearModel, JZS 